\title{
POSITIVELY CURVED MANIFOLDS WITH MAXIMAL DISCRETE SYMMETRY RANK
}

\author{
Fuquan FAnG ${ }^{1} \&$ XiaOchun Rong ${ }^{2}$
}

\begin{abstract}
Let $M$ be a closed simply connected $n$-manifold of positive sectional curvature. We determine its homeomorphism or homotopic type if $M$ also admits an isometric elementary $p$-group action of large rank. Our main results are: There exists a constant $p(n)>0$ such that (1) If $M^{2 n}$ admits an effective isometric $\mathbb{Z}_{p}^{k}$-action for a prime $p \geq p(n)$, then $k \leq n$ and "=" implies that $M^{2 n}$ is homeomorphic to a sphere or a complex projective space. (2) If $M^{2 n+1}$ admits an isometric $S^{1} \times \mathbb{Z}_{p}^{k}$-action for a prime $p \geq p(n)$, then $k \leq n$ and " $=$ " implies that $M$ is homeomorphic to a sphere. (3) For $M$ in (1) or (2), if $n \geq 7$ and $k \geq\left[\frac{3 n}{4}\right]+2$, then $M$ is homeomorphic to a sphere or homotopic to a complex projective space.
\end{abstract}

\section{INTRODUCTION}

A basic problem in Riemannian geometry is the classification of the positively curved manifolds whose isometry groups are large (cf. [Gro]). The largeness is measured by the dimension (resp. the rank of a maximal torus) of the isometry group or the dimension of its orbit space. A typical example is the classification of homogeneous manifolds of positive curvature ([AW], [Be], [Ber], [Wa]).

Recently, there has been considerable progress on the classification of positively curved manifolds with large symmetry rank (cf. [FMR], [FR1,2], [GS], [Hi], [HK], [Ro2], [PS], [Wi], [Ya], etc). The symmetry rank of a Riemannian manifold is the rank of a maximal torus of the isometry group.

The maximal rank theorem of Grove-Searle asserts that if a closed positively curved $n$-manifold $M$ admits an isometric torus $T^{k}$-action, then $k \leq\left[\frac{n+1}{2}\right]$ (the integer part) and "=" if and only if $M$ is diffeomorphic to a sphere, a lens space or a complex projective space ([GS]). Note that any $G$-action considered in this paper is assumed to be effective.

A natural next step is to (homeomorphically) classify closed simply connected positively curved manifolds of the almost maximal symmetry rank $\left[\frac{n+1}{2}\right]-1=\left[\frac{n-1}{2}\right]$. This was previously done for $n=4$ by Hsiang-Kleiner ([HK]), for $n=5$ by Rong ([Ro2]). Recently, the homeomorphism classification for all $n \geq 8$ was obtained by Fang-Rong ([FR1], cf. [FMR], [Wi]). And these manifolds are homeomorphic to a sphere or a complex projective space or a quaternionic projective plane (for

\footnotetext{
${ }^{1}$ Supported by CNPq of Brazil, NSFC Grant 19741002, RFDP and Qiu-Shi Foundation of China ${ }^{2}$ Supported partially by NSF Grant DMS 0203164 and a research found from Capital normal university
} 
$n=8$ ). The situation in the remaining dimensions $n=6,7$ are quite subtle and complicated; there are many examples which are not homeomorphic to any rank one symmetric space ([AW], $[\mathrm{Es}])$. The homeomorphism classification in dimensions 6 and 7 has been under investigation ([FR1,2]).

Recently, Burkhard Wilking obtained the following almost 1/2-maximal rank theorem: For $n \geq 10$, if a closed simply connected positively curved $n$-manifold $M$ with symmetry rank at least $(n / 4+1)$, then $M$ is homeomorphic to a sphere or a quaternionic projective space or homotopy equivalence to a complex projective space $([\mathrm{Wi}])$.

A new tool used in $[\mathrm{Wi}]$ (also in $[\mathrm{FR}]$ ) is a connectedness theorem of Wilking: If $N$ is a closed totally geodesic $r$-submanifold of an $n$-manifold $M$ of positive sectional curvature, then the homotopy group $\pi_{i}(M, N)=0$ for $i \leq 2 r-n+1$ (see Theorem 1.11). Indeed, this theorem, together with other connectedness theorems (see Theorems 1.9-1.12) will play a crucial role in the present paper.

Hopefully, the above classification results together would cover almost all known examples of positively curved manifolds with large symmetry rank, except in dimension 13 ([Ba1]). This should be helpful in looking for new examples of positively curved manifolds (cf. [Gro]).

The purpose of this paper is to study the following more general problem (compare to Problem 24 in [Pe]):

\section{Classify positively curved manifolds which admit isometric $\mathbb{Z}_{p}^{k}$-actions (k large).}

One hopes that this will lead to new insights on positively curved manifolds with abelian symmetry (see Problems 0.1 and 0.2 ).

A guideline is to extend theorems on positively curved manifolds with (large) symmetry rank to positively curved manifolds with (large) discrete symmetry rank. The discrete $p$-symmetry rank ( $p$ is a prime) is the largest number $r$ such that the isometry group contains an elementary $p$-group of rank $r$. This is partly justified by a fascinating fact in the theory of compact transformation groups: a $T^{k}$-action shares many properties with an elementary $p$-group $\mathbb{Z}_{p}^{k}$-action (e.g. the Smith theorem and the Borel theorem) while these properties are generally false for any other compact Lie group (cf. [Hs]).

We begin to state the main results in this paper. The first two results (Theorems A and B) may be considered as an analog of the maximal rank theorem of GroveSearle for elementary $p$-groups.

\section{Theorem A (Maximal Discrete Rank).}

There exists a constant $p(n)>0$ such that if a closed simply connected $2 n$ manifold of positive sectional curvature admits an isometric $\mathbb{Z}_{p}^{k}$-action with prime $p \geq p(n)$, then $k \leq n$ and "=" implies that $M$ is homeomorphic to a sphere or a complex projective space.

The number $p(n)$ can be chosen to be the Gromov's Betti number bound for closed $n$-manifolds of non-negative sectional curvature (see Theorem 1.7).

A basic ingredient in the proof is that the $\mathbb{Z}_{p}^{k}$-fixed point set is not empty; see Lemma 2.1 (note that this may not hold when $p$ is small; the unit sphere $S^{2}$ admits isometric $\mathbb{Z}_{2}^{3}$-action by reflections without fixed point). By comparing this to the Isotropy rank theorem (cf. [Gro]), a natural problem arises: 
Problem 0.1. Let $M$ be a closed (2n+1)-manifold of positive sectional curvature. Show that for $p \geq p(n)$, any isometric $\mathbb{Z}_{p}^{k}$-action $(k \geq 2)$ has a subgroup isomorphic to $\mathbb{Z}_{p}^{k-1}$ whose fixed point set is not empty.

Note that Problem 0.1 implies that the fundamental group of any positively curved $n$-manifold cannot be isomorphic to $\mathbb{Z}_{p} \oplus \mathbb{Z}_{p}$ for $p \geq p(n)$ (compare to [Ba2], [GSh], [Sh]). Indeed, this is also a consequence of the Almost cyclicity conjecture (cf. [Ro1]).

If one solves Problem 0.1, then following the argument in this paper one can prove a Maximal Discrete Rank theorem in odd-dimensions. We will prove the case of Problem 0.1 when $\mathbb{Z}_{p}^{k+1}$ is a subgroup of $S^{1} \times \mathbb{Z}_{p}^{k}$ (see Lemma 3.1). Hence, we are able to prove

\section{Theorem B.}

Let $M$ be a closed simply connected $(2 n+1)$-manifold of positive sectional curvature. If $M$ admits an isometric $S^{1} \times \mathbb{Z}_{p}^{k}$-action $(p \geq p(n))$, then $k \leq n$ and "=" implies that $M$ is homeomorphic to a sphere.

Recall that the maximal rank theorem of Grove-Searle follows easily from the theorem in [GS]: If a closed positively curved manifold $M$ admits an isometric circle action with fixed point set of codimension 2, then $M$ is diffeomorphic to a sphere, a lens space, or a complex projective space. It is easy to see, using [Sm] and the connectedness theorem of Wilking mentioned earlier, that in the above, if one replaces the isometric $S^{1}$-action by any isometric $\mathbb{Z}_{p}$-action, then the universal covering of $M$ is homeomorphic to a sphere, provided $\operatorname{dim}(M)$ is odd (see Lemma $4.2)$.

Problem 0.2. Let $M$ be closed simply connected $2 n$-manifold of positive sectional curvature. Assume $M$ admits an isometric $\mathbb{Z}_{p}$-action $(p \geq p(n))$ with a fixed point set of codimension 2. Show that $M$ is homotopic to a sphere or a complex projective space.

Next, we consider the problem of a possible analog of Wilking's almost $1 / 2$ maximal rank theorem for the elementary $p$-groups. This is quite subtle due to that the almost 1/2-maximal discrete symmetry rank seems to be an inadequate condition for the existence of a totally geodesic submanifold of codimension at most $\operatorname{dim}(M) / 4$ (cf. [Wi]). Nevertheless, a discrete analog of Wilking's almost $1 / 2$-maximal rank theorem would be a $c$-maximal discrete rank theorem for some constant $\frac{1}{2} \leq c<1$. Our last two theorems imply that $c$ is not greater than $3 / 4$.

\section{Theorem C (Almost 3/4-maximal Discrete Rank).}

For $n \geq 7$, if a closed simply connected $2 n$-manifold of positive sectional curvature satisfies

$$
\text { Symrank }_{p}(M) \geq\left\{\begin{array}{ll}
{\left[\frac{3 n}{4}\right]+2} & n=1,2 \bmod 4 \\
{\left[\frac{3 n}{4}\right]+1} & \text { otherwise }
\end{array} \quad p \geq p(n),\right.
$$

then $M$ is homeomorphic to a sphere or homotopic equivalent to a complex projective space.

The following is a weak version of Theorem $\mathrm{C}$ in odd-dimensions; which also partially strengthens Theorem B. 


\section{Theorem D.}

For $n \geq 7$, a closed simply connected $(2 n+1)$-manifold of positive sectional curvature is homeomorphic to a sphere, if $M$ admits an isometric $S^{1} \times \mathbb{Z}_{p}^{k}$-action $(p \geq p(n))$ such that $k \geq\left\{\begin{array}{ll}{\left[\frac{3 n}{4}\right]+2} & n=1 \bmod 4 \\ {\left[\frac{3 n}{4}\right]+1} & \text { otherwise }\end{array}\right.$.

Remark 0.3. Note that in cases of Theorems C and D where there is an isometric $T^{k}$-action (resp. $T^{k+1}$-action), the lower bound for $k$ can be further lowered by one, using the equivariant version of Theorem 1.11 in $[\mathrm{Wi}]$.

We now give an indication about the proof of Theorem A (the proofs of Theorems $\mathrm{C}$ and $\mathrm{D}$ are in a similar spirit but are more involved).

The proof is based on the following deep topological results: For $n \geq 4$ the generalized Poincare conjecture holds true ( $n=4$ by Freedman and $n \geq 5$ by Smale, $[\mathrm{Fr}],[\mathrm{Sm}]$ ) and the theory of Sullivan's characteristic varieties [Su] (cf. Theorem 1.3), which implies that a homotopy complex projective space $M$ is homeomorphic to $\mathbb{C} P^{n}$ if $M$ contains a closed submanifold of codimension 2 dual to a generator of $H^{2}(M ; \mathbb{Z}) \cong \mathbb{Z}$ which is homeomorphic to $\mathbb{C} P^{n-1}$ (see Lemma 2.4).

Consider $M$ as in Theorem A. By employing the Borel theorem (cf. [Hs]) and the Gromov's Betti number estimate $([\mathrm{Gr}])$, we show that for $p \geq p(n)$, the $\mathbb{Z}_{p}^{k}$-fixed point set is not empty (Lemma 2.1). From the isotropy representation at a fixed point, it follows that $k \leq n$, and "=" implies that there is a sequence of closed totally geodesic submanifolds,

$$
M^{4} \subset M^{6} \subset \cdots \subset M^{2 n}=M,
$$

such that $M^{2 i}$ admits an isometric $\mathbb{Z}_{p}^{i}$-action. By the theorem of Wilking mentioned earlier (Theorem 1.11), it is easy to see that if $M^{4}$ is homotopic to a sphere or a complex projective space, then $M$ is homotopic to a sphere or a complex projective space. Indeed, if $M^{4}$ is homeomorphic to a sphere or complex projective space, then by the above topological results one upgrades the homotopy equivalence to that $M$ is homeomorphic to a sphere or a complex projective space. We then complete the proof by showing that $M^{4}$ is homeomorphic to a sphere or a complex projective space (Proposition 2.3).

The rest of the paper is organized as follows:

In Section 1, we collect results that are used in the proofs of Theorems A-D.

In Section 2, we prove Theorem A.

In Section 3, we prove Theorem B.

In Section 4, we prove Theorems $\mathrm{C}$ and $\mathrm{D}$.

Acknowledgment: The authors would like to thank Burkhard Wilking for some useful comments on the early version and thank Wolfgang Ziller for some references. The second author would like to thank Burkhard Wilking for informing him of the main results in $[\mathrm{Wi}]$.

\section{PRELIMinaRies}

In this section, we will collect theorems that will be used in the rest of the paper. 


\section{a. Some deep topological theorems.}

The generalized Poincaré conjecture says that any homotopy $n$-sphere is a homeomorphic sphere. By $[\mathrm{Fr}]$ and [Sm], this conjecture remains open only for $n=3$.

Theorem 1.1 (Freedman). Every homotopy 4-sphere is homeomorphic to a sphere.

Theorem 1.2 (Smale). For $n \geq 5$, every homotopy $n$-sphere is homeomorphic to a sphere.

In general, the homotopy class of a closed manifold may contain many homeomorphism types. In [Su], Sullivan classified the homeomorphic types of a homotopy complex projective space.

Let $M$ be a manifold with the homotopy type of $\mathbb{C} P^{n}$. Let $h: M \rightarrow \mathbb{C} P^{n}$ be a homotopy equivalence. For any $\mathbb{C} P^{i} \subset \mathbb{C} P^{n}$, let $h^{-1}\left(\mathbb{C} P^{i}\right) \subset M$ denote the transverse submanifold (the preimage of a map homotopic to $h$ and transverse to $\mathbb{C} P^{i}$.) Let $\sigma_{h}(i)$ be the signature (an integer) of $h^{-1}\left(\mathbb{C} P^{i}\right)$ if $i$ is even (an integer), and the Kervaire invariant (a mod 2 integer) if $i$ is odd (cf. [Su]). The invariant $\sigma_{h}(i)$ depends only on the homotopy class $h$. Indeed, if $N^{2 i} \subset M$ is another manifold homologous to $h^{-1}\left(\mathbb{C} P^{i}\right)$, then $\sigma_{h}(i)$ is equal to the signature (resp. Kervaire invariant) of $N^{2 i}$.

Theorem 1.3 (Sullivan). Let $h S\left(\mathbb{C} P^{n}\right)$ denote the set of closed manifolds homotopy equivalent to $\mathbb{C} P^{n}$. For $i=1, \cdots,[n / 2], \sigma_{h}(i)$ defines an one-to-one correspondence between $h S\left(\mathbb{C} P^{n}\right)$ and the set $\prod_{i=1}^{[n / 4]}\left(\mathbb{Z} \times \mathbb{Z}_{2}\right)$.

Recall that the Kervaire invariant is zero if a manifold of dimension $(4 i+2)$ has no middle dimensional homology, e.g. manifolds homotopy equivalent to $\mathbb{C} P^{2 i+1}$. On the other hand, the signature is a homotopy invariant. As an immediate corollary of Theorem 1.3, one gets

Corollary 1.4. If $M \in h S\left(\mathbb{C} P^{n}\right)$ has a codimension 2 submanifold homeomorphic to $\mathbb{C} P^{n-1}$ which represents a generator of $H_{2 n-2}(M)$, then $M$ is homeomorphic to $\mathbb{C} P^{n}$.

\section{b. Fixed point sets.}

Let $G$ denote a compact Lie group. For a $G$-space, let $F(G, M)$ denote the fixed point set. Given any $G$-invariant metric, each component of $F(G, M)$ is a totally geodesic submanifold.

It is well known that for $G=T^{k}$ or $\mathbb{Z}_{p}^{k}$ ( $p$ is a prime), the topology of $F(G, M)$ is closely related the topology of $M$. For instance, the Euler characteristic $\chi(M)=$ $\chi\left(F\left(T^{k}, M\right)\right)$. In the proof of Theorem A, the following results will be used (cf. $[\mathrm{Hs}])$.

Lemma 1.5. Let $M$ be a closed $\mathbb{Z}_{p}$-space ( $p$ is a prime).

Then

$$
\chi(M)=\chi\left(F\left(\mathbb{Z}_{p}, M\right)\right)(\bmod p) .
$$


Theorem 1.6 (Borel). Let $G=T^{k}$ or $\mathbb{Z}_{p}^{k}$ ( $p$ is a prime), and let $M$ be a $G$-space. Then

$$
\operatorname{rank}\left(H_{*}(F(G, M), \ell)\right) \leq \operatorname{rank}\left(H_{*}(M, \ell)\right),
$$

where $\ell=\mathbb{R}$ if $G=T^{k}$ and $\mathbb{Z}_{p}$ otherwise.

By Theorem 1.6, the number of components of $F(G, M)$ is bounded above by the total Betti number of $M$. If $M$ also has non-negative sectional curvature, then Gromov Betti number estimate $([\mathrm{Gr}])$ implies that the number of components of $F(G, M)$ is bounded above a constant depending only on $\operatorname{dim}(M)$.

Theorem 1.7 (Gromov). Let $M$ be a closed $n$-manifold of non-negative sectional curvature. Then for any coefficient field $\ell$, the total Betti number, $\operatorname{rank}\left(H_{*}(M, \ell)\right) \leq$ $b(n)$.

\section{c. The connectedness principle of positive curvature.}

As seen in the introduction, in the study of positively curved manifolds with large isometry groups, the recent break-through is the Wilking's connectedness theorem (see Theorem 1.11). His proof is a combination of the Morse theory and the standard Synge type argument. Soon after Wilking announced the results of [Wi], a connectedness principle of positive curvature was obtained in [FMR] (see Theorem 1.8) which not only gives a uniform formulation for the Synge theorem (Theorem 1.12), the Frankel theorem (Theorem 1.9) and the Wilking theorem (Theorem 1.11), but also includes a new regularity theorem (which is not required in this paper). The connectedness principle of positive curvature can be viewed as an analog of the connectedness principle in the algebraic geometry, for details, see [FMR] and references within.

An immersion is called totally geodesic if the second fundamental form vanishes.

Theorem 1.8 (Connectedness Principle of positive curvature). Let $M$ be a closed m-manifold of positive sectional curvature, and let $\Delta$ denote the diagonal of $M \times M$. Assume $N=N_{1} \times N_{2}$ and $f=\left(f_{1}, f_{2}\right): N \rightarrow M \times M$, where $f_{i}: N_{i} \rightarrow M$ is totally geodesic $n_{i}$-dimensional immersion. Then $\left(n=n_{1}+n_{2}\right)$

(1.8.1) If $n \geq m$, then $f^{-1}(\Delta)$ is non-empty.

(1.8.2) If $n \geq m+1$ and $M$ is simply connected, then $f^{-1}(\Delta)$ is connected.

(1.8.3) For $n \geq m+i$ there is an exact sequence

$$
\pi_{i}\left(f^{-1}(\Delta)\right) \rightarrow \pi_{i}(N) \stackrel{\left(p_{1} f\right)_{*}-\left(p_{2} f\right)_{*}}{\longrightarrow} \pi_{i}(M) \rightarrow \pi_{i-1}\left(f^{-1}(\Delta)\right) \rightarrow \cdots .
$$

(1.8.4) There are natural isomorphisms, $\pi_{i}\left(N_{1}, f^{-1}(\Delta)\right) \rightarrow \pi_{i}\left(M, N_{2}\right)$ and $\pi_{i}\left(N_{2}, f^{-1}(\Delta)\right) \rightarrow$ $\pi_{i}\left(M, N_{1}\right)$ for $i \leq n-m$ and surjections for $i=n-m+1$, where $\pi_{i}\left(N_{j}, f^{-1}(\Delta)\right)$ is understood as the $i$-th homotopy group of the composition map $f^{-1}(\Delta) \subset N \stackrel{p_{j}}{\longrightarrow} N_{j}$.

Note that (1.8.1) is a strengthened version of the Frankel theorem (stated below) where embedded submanifolds are assumed.

Theorem 1.9 (Frankel). Let $M$ be a closed manifold of positive sectional curvature, and let $f_{i}: N_{i} \rightarrow M$ be two closed totally geodesic immersions. If $\operatorname{dim}\left(N_{1}\right)+$ $\operatorname{dim}\left(N_{2}\right) \geq \operatorname{dim}(M)$, then $f_{1}\left(N_{1}\right) \cap f_{2}\left(N_{2}\right) \neq \emptyset$.

Note that (1.8.4) immediately implies the following: 
Theorem 1.10. Let $M$ be a closed m-manifold of positive curvature. Let $N_{1}, N_{2}$ be embedded totally geodesic submanifolds in $M$ of dimensions $n_{1}, n_{2}$ respectively. Set $n=n_{1}+n_{2}$. Then there are natural isomorphisms

$$
\pi_{i}\left(N_{1}, N_{1} \cap N_{2}\right) \rightarrow \pi_{i}\left(M, N_{2}\right), \quad \pi_{i}\left(N_{2}, N_{1} \cap N_{2}\right) \rightarrow \pi_{i}\left(M, N_{1}\right)
$$

for $i \leq n-m$, and surjections for $i=n-m+1$.

A map from $N$ to $M$ is called $(i+1)$-connected, if it induces an isomorphism up to the $i$-th homotopy group and a surjective homomorphism on the $(i+1)$-th homotopy group.

Theorem 1.10 implies the following result of Wilking ([FMR]).

Theorem 1.11 (Wilking). Let $M$ be a closed m-manifold of positive sectional curvature. Let $N_{1}$ and $N_{2}$ be two totally geodesic submanifolds of dimensions $n_{1}$ and $n_{2}$. If $n_{2} \geq n_{1}$, then the inclusions, (1.11.1) $i_{2}: N_{2} \hookrightarrow M$ is $\left(2 n_{2}-m+1\right)$-connected.

(1.11.2) $N_{1} \cap N_{2} \hookrightarrow N_{1}$ is $\left(n_{1}+n_{2}-m\right)$-connected.

Indeed, the classical Synge theorem can also be formulated as a connectedness theorem (compare (1.8.1)). This was pointed to us by Karsten Grove.

Theorem 1.12 (Synge). Let $M$ be a closed orientable $n$-manifold of positive sectional curvature, and let $\phi$ be an isometry. Let $f: M \rightarrow M \times M$ be a totally geodesic embedding by $f(x)=(x, \phi(x))$. Then $f^{-1}(\Delta)$ is not empty under the following situations:

(1.12.1) $n$ is even and $\phi$ is orientation preserving.

(1.12.2) $n$ is odd and $\phi$ is orientation reversing.

There are other connectedness theorems that follow from Theorem 1.8 (see $[\mathrm{FMR}]$ ); we do not state them here since they are not required in this paper.

\section{d. Alexandrov spaces with positive curvature.}

Recall that an Alexandrov space, $X$, is a finite Hausdorff dimensional complete metric space with a lower curvature bound in distance comparison sense, cf. [BGP]. In particular, a Riemannian manifold of sectional curvature bounded from below is an Alexandrov space. For an Alexandrov space, one can define orientability in a standard way using atlas of distance maps; see [Pet].

The following version of Theorem 1.12 for Alexandrov spaces of positive curvature will be used in the proof of Theorem B.

Theorem 1.13 (Petrunin). Let $X$ be an orientable Alexandrov space of $\operatorname{cur}(X) \geq$ 1. If $\operatorname{dim}(X)$ is even, then any orientation-preserving isometry has a fixed point. In particular, $X$ is simply connected.

Recall that the quotient space of a Riemannian manifold by an isometric group action is, with the quotient metric, not necessarily a Riemannian manifold (it may not even be a manifold). In the comparison, an advantage with an Alexandrov space is (cf. $[\mathrm{BGP}])$ : 
Lemma 1.14. Let $X$ be an Alexandrov space with curvature $\geq-\Lambda$. Let $G$ be a compact group of isometries. Then, the quotient space, $X / G$, is also an Alexandrov space with curvature $\geq-\Lambda$.

\section{Proof of Theorem A}

For an isometric $T^{k}$-action on a closed positively curved $n$-manifold, a basic property is the Isotropy rank theorem: If $n$ is even, then the fixed point set is not empty, and if $n$ is odd, then there is a circle orbit (cf. [Ko], [Ro2]). This also plays a role in the proof of the maximal rank theorem of Grove-Searle ([GS]).

The following result may be considered as a discrete version of the Isotropy rank theorem in even-dimensions (compare to Lemma 3.1).

Lemma 2.1 (Discrete Isotropy Rank). Assume a closed positively curved $2 n$ manifold $M$ admits an isometric $\mathbb{Z}_{p}^{k}$-action with $p \geq p(n)$. Then the fixed point set is not empty and $k \leq n$ and '=' implies that the fixed point set is finite.

Note that the requirement for a lower bound on $p$ is of necessary; for instance the unit sphere $S^{2 n}$ admits the obvious isometric $\mathbb{Z}_{2}^{2 n+1}$-action without fixed point.

Proof. Without loss of the generality, we may assume that $p(n)>2$. We shall determine the value of $p(n)$ at the end of the proof.

We first assume that $M$ is orientable. Let $H_{p}$ denote any $\mathbb{Z}_{p}$ subgroup of $\mathbb{Z}_{p}^{k}$ generated by $\alpha$. Note that $\alpha^{2}$ is always orientation-preserving and $F\left(H_{p}, M\right)=$ $F\left(\alpha^{2}, M\right)$ since $H_{p}$ is generated by $\alpha^{2}$. By Theorem 1.12 , we can assume an $H_{p^{-}}$ fixed point component $F_{0} \neq \emptyset$. Note that $F_{0}$ is a closed totally geodesic submanifold of even-codimension. We claim that $F_{0}$ is preserved by $\mathbb{Z}_{p}^{k}$. Then, by induction on $n$ one can conclude that $\mathbb{Z}_{p}^{k}$ has a fixed point in $F_{0}$. If not, $F\left(H_{p}, M\right)$ must have at least $p$ components. By Theorem 1.6 and Theorem 1.7, we then obtain

$$
\begin{aligned}
p & \leq \#\left\{\text { components of } F\left(\mathbb{Z}_{p}, M\right)\right\} \\
& \leq \operatorname{rank}\left(H_{*}\left(F\left(\mathbb{Z}_{p}, M\right), \mathbb{Z}_{p}\right)\right) \\
& \leq \operatorname{rank}\left(H_{*}\left(M, \mathbb{Z}_{p}\right)\right) \leq b(n) .
\end{aligned}
$$

By now it is clear that if one chooses $p(n)=b(n)+1$, we then see a contradiction.

Let $F$ be a $\mathbb{Z}_{p}^{k}$-fixed point component. Then $\operatorname{dim}(F)=2(n-r)$ where $r$ is a positive integer. Then, $\mathbb{Z}_{p}^{k}$ acts on the normal space of $F$ at $x$ by isometries. We can consider $\mathbb{Z}_{p}^{k}$ as a subgroup of the maximal torus of $O(2 r)$ and therefore $k \leq r \leq n$ and $k=n$ implies that $r=n$ and thus $\operatorname{dim}(F)=0$.

If $M$ is not orientable, then the double covering $\tilde{M}$ of $M$ is simply connected (Theorem 1.12). Hence, we can apply the above to the lifting $\mathbb{Z}_{p}^{k}$ action on $\tilde{M}$ and conclude the desired result.

The maximality has the following property.

Lemma 2.2. Let the assumptions be as in Theorem $A$ with $k=n$. Then there are closed totally geodesic submanifolds, $M^{4} \subset M^{6} \subset \cdots \subset M^{2 n}=M$ such that $M^{2 i}$ admits an isometric $\mathbb{Z}_{p}^{i}$-action. 
Proof. Consider the linear isotropy representation of $\mathbb{Z}_{p}^{n}$ at a fixed point $x \in M$. Let $\mathbb{Z}_{p}^{n-i} \subset \mathbb{Z}_{p}^{n}$ be the product subgroup of the first $(n-i)$-factors. Let $M^{m_{i}}$ be the connected fixed point submanifold of $\mathbb{Z}_{p}^{n-i}$ containing $x$. It suffices to verify the dimension(s) of the fixed point manifolds are exactly $2 i$.

Note that the tangent space of $M^{m_{i}}$ at $x$ is the invariant linear subspace of the $\mathbb{Z}_{p}^{n-i}$-action on $T_{x} M$. By the proof of Lemma 2.1 we know that $m_{i} \leq 2 i$. Consider the action of $\mathbb{Z}_{p}^{n}$ on $F_{i}$. This action has the principal isotropy group $\mathbb{Z}_{p}^{n-i}$. Therefore the induced action by the quotient group $\mathbb{Z}_{p}^{i}=\mathbb{Z}_{p}^{n} / \mathbb{Z}_{p}^{n-i}$ is effective. This implies that $\mathbb{Z}_{p}^{i}$ embeds into the orthogonal group $O\left(m_{i}\right)$ as a subgroup. Therefore $m_{i} \geq 2 i$. The desired result follows.

We first prove Theorem A for $n=4$. For a closed manifold $M$, let $\chi(M)$ denote the Euler characteristic of $M$.

Proposition 2.3. Let $M^{4}$ be a closed simply connected manifold of positive sectional curvature. If $M^{4}$ admits an isometric $\mathbb{Z}_{p}^{2}$-action with $p>\chi\left(M^{4}\right)$, then $M^{4}$ is homeomorphic to $S^{4}$ or $\mathbb{C} P^{2}$.

Note that one can alway apply Proposition 2.3 if $p>b(4)$, the constant from Theorem 1.7 for $n=4$.

Proof of Proposition 2.3. We first show that the fixed point set $F\left(\mathbb{Z}_{p}^{2}, M^{4}\right) \neq \emptyset$. Since $F\left(\mathbb{Z}_{p}^{2}, M^{4}\right)$ has even codimension, from the isotropy representation $F\left(\mathbb{Z}_{p}^{2}, M^{4}\right)$ must be a finite set.

We will argue by contradiction. By Theorem 1.12, we can assume a $\mathbb{Z}_{p}$-subgroup, $H_{p}$, of $\mathbb{Z}_{p}^{2}$ such that $F\left(H_{p}, M^{4}\right) \neq \emptyset$. If $\operatorname{dim}\left(F\left(H_{p}, M^{4}\right)\right)=2$, then $F\left(H_{p}, M^{4}\right)$ has only one component of dimension 2 and therefore $\mathbb{Z}_{p}^{2}$ must preserve this component (see Theorem 1.9). Since the component is a closed totally geodesic submanifold (which is homeomorphic to $S^{2}$ ), it contains $\mathbb{Z}_{p}^{2}$-fixed points; a contradiction. If $F\left(H_{p}, M^{4}\right)$ is a finite set, since $F\left(\mathbb{Z}_{p}^{2}, M^{4}\right)=\emptyset,\left|F\left(H_{p}, M^{4}\right)\right| \geq p$. By Theorem 1.6, we obtain

$$
p \leq \operatorname{rank}\left(H_{*}\left(F\left(H_{p}, M^{4}\right), \mathbb{Z}_{p}\right) \leq \operatorname{rank}\left(H_{*}\left(M^{4}, \mathbb{Z}_{p}\right)\right)=\chi\left(M^{4}\right)<p,\right.
$$

a contradiction.

We then show that $\chi\left(M^{4}\right) \leq 3$. By $[\mathrm{Fr}]$, it follows that $M^{4}$ is homeomorphic to $S^{4}$ or $\mathbb{C} P^{2}$.

Observe that at each $\mathbb{Z}_{p}^{2}$-fixed point, from the isotropy representation we see that there are two $\mathbb{Z}_{p}$-isotropy groups whose fixed point sets are two spheres and their intersection is this $\mathbb{Z}_{p}^{2}$-fixed point. Hence, we can use a one-dimensional complex to represent the singular set in the orbit space $M^{4} / \mathbb{Z}_{p}^{2}$, where every edge indicates a two sphere fixed point set of some isotropy group. The $\mathbb{Z}_{p}^{2}$-fixed point are the vertices and at each vertex there are two edges. If there are more than three vertices, then there must exist two edges which do not share any vertex. This implies that there are two totally geodesic 2 -spheres in $M^{4}$ which do not intersect, a contradiction (see Theorem 1.9). On the other hand, the number of vertices cannot be one.

By Lemma 1.5, the above implies that $\chi\left(M^{4}\right)=2+k p<p$ or $3+k p<p$ with $k \geq 0$ since $\chi\left(M^{4}\right)=2+b_{2} \geq 2$. This implies that $\chi\left(M^{4}\right)=2$ or 3 . 
Lemma 2.4. Let $M$ be a homotopy complex projective space. If $M$ has a codimension 2 submanifold $N$ homeomorphic to $\mathbb{C P}^{n-1}$ such that the inclusion map is a 3-connected, then $N$ represents a generator of $H_{2 n-2}(M)$ and therefore $M$ is homeomorphic to $\mathbb{C} P^{n}$.

Proof. By Corollary 1.4, it suffices to show that $N$ represents a generator of $H_{2 n-2}(M)$. Let $x \in H^{2}(M)$ denote a generator. Recall that the cohomology $\operatorname{ring} H^{*}(M) \cong$ $H^{*}\left(\mathbb{C} P^{n}\right) \cong \mathbb{Z}[x] /\left(x^{n+1}=0\right)$. Since $i$ is a 3 -equivalence, $i: N \rightarrow M$ induces an isomorphism $i^{*}: H^{2}(M) \rightarrow H^{2}(N)$. If $i_{*}([N]) \in H_{2 n-2}(M)$ represents $d$ times the generator, then $i^{*}\left(x^{n-1}\right)[N]=d$. On the other hand, since the cohomology ring $H^{*}(N)=\mathbb{Z}\left[i^{*}(x)\right] /\left(i^{*}(x)^{n}=0\right)$. Thus $i^{*}\left(x^{n-1}\right)[N]= \pm 1$. This proves that $d= \pm 1$, i.e. $i_{*}([N])$ is a generator of $H_{2(n-1)}(M)$.

With the above preparation, we are ready to prove Theorem A.

Proof of Theorem A.

We first show that $M$ is homotopy equivalent to a sphere or a complex projective space.

By Lemma 2.1, we obtain $k \leq n$. By Lemma 2.2, we obtain a sequence of closed orientable totally geodesic submanifolds,

$$
M^{4} \subset M^{6} \subset \cdots \subset M^{2 n}=M .
$$

By Theorem 1.11, we orderly conclude that $M^{2 n-2}, \ldots, M^{4}$ are simply connected. By Proposition 2.3, we conclude that $M^{4}$ is homeomorphic to $S^{4}$ or $\mathbb{C} P^{2}$. By Theorem 1.11 again, we conclude that $M^{6}$ satisfies that $\pi_{2}\left(M^{6}\right)=\mathbb{Z}$ (resp. 0 ) if $M^{4}=\mathbb{C} P^{2}$ (resp. $\left.S^{4}\right)$ and $\pi_{3}(M)=0$. By the Poincaré duality and the Hurewicz theorem, we conclude that $M^{6}$ is homotopy equivalent to $\mathbb{C} P^{3}$ (resp. $\left.S^{6}\right)$ if $M^{4} \stackrel{\text { homeo }}{\simeq} \mathbb{C} P^{2}$ (resp. $\left.S^{4}\right)$. By iterating the same argument to $M^{8}, \ldots, M^{2 n}$, we conclude that $M^{2 n}$ is homotopic to a sphere or a complex projective space.

Finally, if $M$ is a homotopy sphere, then by Theorems 1.1 and $1.2 M$ is a homeomorphic sphere for $n \geq 4$.

If $M$ is a homotopy complex projective space, by Proposition 2.3, Lemma 2.4 and Theorem 1.11 we then conclude that $M^{6}$ is homeomorphic to a complex projective space. Iterating this $(n-1)$-times, we eventually conclude that $M^{2 n}=M$ is homeomorphic to a complex projective space.

\section{Proof of Theorem B}

The proof of Theorem B follows the same strategy as in the proof of Theorem A.

The first lemma may be considered as a weak version of Lemma 2.1 in odddimensions.

Lemma 3.1. Let $M$ be a closed simply connected $(2 n+1)$-manifold of positive sectional curvature. If $M$ admits an isometric $S^{1} \times \mathbb{Z}_{p}^{k}$-action $(p \geq p(n))$ then $k \leq n$ and $\mathbb{Z}_{p}^{k+1} \subset S^{1} \times \mathbb{Z}_{p}^{k}$ has a $\mathbb{Z}_{p}^{k}$-subgroup whose fixed point set is not empty and contains a circle orbit, and ' $=$ ' implies that the fixed point set has dimension one. 
Sublemma 3.2. Let the assumptions be as in Lemma 3.1. Then the $S^{1} \times \mathbb{Z}_{p}^{k}$-action has a finite isotropy group.

Proof. Let $p: M \rightarrow M^{*}:=M / S^{1}$ denote the orbit projection, where $S^{1}=S^{1} \times$ \{id\} $\subset S^{1} \times \mathbb{Z}_{p}^{k}$. Since $M$ is orientable and since the $S^{1}$-action preserves the orientation, $M^{*}$ is an orientable Alexandrov space of positive curvature (Lemma 1.14). Note that the $\mathbb{Z}_{p}^{k}$-action on $M$ descends to an effective $\mathbb{Z}_{p}^{k}$-action on $M^{*}$.

Let $H \cong \mathbb{Z}_{p}$ be any subgroup of $\mathbb{Z}_{p}^{k}$. By Theorem 1.13 we can assume that $H$ has a fixed point $x^{*} \in M^{*}$. Let $S^{1}(x)$ denote the $S^{1}$-orbit at $x$ such that $p(x)=x^{*}$. Then $S^{1} \times H$ preserves $S^{1}(x)$ (which is either a circle or a point). This implies that the isotropy group $H_{x}$ (of the $S^{1} \times \mathbb{Z}_{p}^{k}$-action) at $x$ contains a subgroup isomorphic to $H$.

If $S^{1}(x)$ is a circle, then the isotropy group of $S^{1} \times \mathbb{Z}_{p}^{k}$ at $x$ is finite. Otherwise, $H_{x}$ contains $S^{1} \times H$. Consider the isotropy representation of $H_{x}$ at the tangent space at $x$. From standard linear algebra, one concludes that $H_{x}$ has a finite isotropy group on the tangent space.

Proof of Lemma 3.1.

We proceed by induction on $n, \operatorname{dim}(M)=2 n+1$. Note that Lemma 3.1 holds trivially for $n=1$. Assume Lemma 3.1 holds for $\operatorname{dim}(M)<2 n+1$.

By Sublemma 3.2, we can assume that the $S^{1} \times \mathbb{Z}_{p}^{k}$-action has a finite isotropy group $H$. Let $F$ be a component of $F(H, M)$ which contains a point $x$ whose isotropy group is $H$ (note that this may not hold for every component of $F(H, M)$ ). We first claim that $S^{1} \times \mathbb{Z}_{p}^{k}$ preserves $F$. Since the $S^{1}$-subgroup always preserves $F$, it suffices to show that $\mathbb{Z}_{p}^{k}$ preserves $F$. Otherwise, since $\mathbb{Z}_{p}^{k}$ preserves $F(H, M)$, then $F(H, M)$ must contain at least $p$ components. Following the same arguments as in the proof of Lemma 2.1, one obtains a contradiction.

Put $S^{1} \times \mathbb{Z}_{p}^{r}=\left(S^{1} \times \mathbb{Z}_{p}^{k}\right) / H$. Since $x \in F, S^{1} \times \mathbb{Z}_{p}^{r}$ acts effectively on $F$. Note that $F$ is a closed totally geodesic submanifold of odd-dimension $<2 n+1$. If $r=0$, then the proof is complete. If $r>0$, then we can apply the inductive assumption to $\left(F, S^{1} \times \mathbb{Z}_{p}^{r}\right)$ and conclude that $S^{1} \times \mathbb{Z}_{p}^{r}$ has a subgroup $\mathbb{Z}_{p}^{r}$ whose fixed point set in $F$ is not empty. This implies that $H \cong \mathbb{Z}_{p}^{k-r}$ and thus the $S^{1} \times \mathbb{Z}_{p}^{k}$ has a $\mathbb{Z}_{p}^{k}$-subgroup whose fixed point set is not empty.

Finally, if $F_{0}$ is a $\mathbb{Z}_{p}^{k}$-fixed point component, then $\mathbb{Z}_{p}^{k}$ acts effectively on a normal sphere space to $F_{0}$. If $\operatorname{dim}\left(F_{0}\right)=2 r+1$, then from the proof of Lemma $2.2 k \leq n-r$ and thus $k \leq n$. If $k=n$, then $r=0$ and thus $\operatorname{dim}\left(F_{0}\right)=1$.

Lemma 3.3. Let the assumptions be as in Theorem $B$ with $k=n$. Then there are closed totally geodesic submanifolds, $M^{3} \subset M^{5} \subset \cdots \subset M^{2 n+1}=M$ such that $M^{2 i+1}$ admits an isometric $\mathbb{Z}_{p}^{i}$-action.

Proof. By Lemma 3.1, we can assume a subgroup $H \cong \mathbb{Z}_{p}^{n}$ whose fixed point set is a circle. Consider the isotropy representation of $H$ on the normal space of the circle. Note that the normal space has dimension $2 n$. By now the rest of the proof follows exactly the same argument as in the proof of Lemma 2.2 .

Proof of Theorem B.

By Theorems 1.2, it suffices to show that $M$ is a homotopy sphere (note that for $n=1$, one may use $[\mathrm{Ha}])$. 
By Lemma 3.3, we obtain a sequence closed orientable totally geodesic submanifolds,

$$
M^{3} \subset M^{5} \subset \cdots \subset M^{2 n+1} .
$$

By Theorem 1.11, we conclude in order that $M^{2 n-1}, \ldots, M^{3}$ are simply connected. Note that $M^{3}$ is a homotopy sphere. By Theorem 1.11 again, we then conclude that $M^{5}$ is 2-connected and thus a homotopy sphere (by the Poincaré duality and the Hurewicz theorem). By iterating the same argument to $M^{7}, \ldots, M^{2 n+1}$, we eventually conclude that $M^{2 n+1}$ is homotopic to a sphere.

\section{Proofs of Theorems C And D}

First, we need some preparation.

Lemma 4.1. Let $M$ be a closed simply connected 2 -manifold of positive sectional curvature. If there is a closed submanifold $N$ of dimension at least $(n+1)$ such that $N$ is homotopic equivalent to a sphere or a complex projective space and the inclusion $i: N \hookrightarrow M^{2 n}$ is at least an n-equivalence, then $M$ is homotopy equivalent to a sphere or a complex projective space.

Proof. Observe that if $N$ is homotopic to a sphere, then by the Poincaré duality $M$ is a homotopy sphere. Hence, we can assume that $N$ is homotopic to a complex projective space. Since $\pi_{1}(M)=1$, it suffices to show that the cohomology ring $H^{*}(M)$ is isomorphic to the truncated polynomial ring $\mathbb{Z}[x] /\left(x^{n+1}=0\right)$ for a generator $x \in H^{2}(M)$.

Let $x \in H^{2}(M)$ be a generator. Note that $\pi_{n}(N)=0$. It is easy to see that $H^{*}(M)$ is isomorphic to $\mathbb{Z}$ up to degree $n$.

If $n$ is even, by the Poincaré duality $\left(x^{\frac{n}{2}}\right)^{2}[M]= \pm 1$ and thus $H^{*}(M)$ is isomorphic to $\mathbb{Z}[x] /\left(x^{n+1}=0\right)$.

If $n$ is odd, write $x^{\frac{n+1}{2}}=d y$ for some integer $d$, where $y \in H^{n+1}(M) \cong \mathbb{Z}$ is a generator. Since $i^{*}(x)$ is a generator of $H^{2}(N)$, the above implies that $d i^{*}(y)=$ $\left(i^{*}(x)\right)^{\frac{n+1}{2}}$ is a generator of $H^{n+1}(N) \cong \mathbb{Z}$ and thus $d= \pm 1$. Consequently, $H^{*}(M)$ is also isomorphic to $\mathbb{Z}[x] /\left(x^{n+1}=0\right)$.

The following lemma was pointed to us by Burkhard Wilking.

Lemma 4.2. Let $M$ be closed simply connected $m$-manifold of positive sectional curvature. Assume that

(4.2.1) If $m=2 n+1$, there is a closed totally geodesic submanifold $N$ of codimension 2 or

(4.2.2) If $m=2 n$, there are two closed totally geodesic submanifolds $N_{1}, N_{2}$ of codimenison 2 such that $\operatorname{dim}\left(N_{1} \cap N_{2}\right)=m-4>0$.

Then $M$ is homotopy equivalent to a sphere or a complex projective space.

Note that (4.2.1) also gives an alternative proof of Theorem B.

Proof. (4.2.1) By [Ha], we can assume $n>2$. By Theorem 1.2, it suffices to show that $M$ is a homotopy sphere. By (1.11.1) the inclusion $i: N \rightarrow M$ is $(2 n-2)$ connected. In particular, $N$ is simply connected. By the Poincaré duality, $H^{1}(N)=$ 
$H_{2 n-2}(N)=0$ and thus $H^{3}(M)=H_{2 n-2}(M)=0$. Thus $H^{2 n-4}(N)=H_{3}(N)=0$ if $m \geq 3$. This implies that $H^{5}(M)=H_{2 n-4}(M)=0$.

By repeating this process one conclude that both $M$ and $N$ are homotopy spheres.

(4.2.2) Set $N=N_{1} \cap N_{2}$. Then $\operatorname{dim}(N)=2 n-4>0$. By Theorem 1.11, $N_{1} \hookrightarrow M$ is $(2(n-2)+1)$-connected. By Lemma 4.1 , it suffices to show that the cohomology ring $H^{*}\left(N_{1}\right)$ is isomorphic to $H^{*}\left(S^{2 n-2}\right)$ or $\mathbb{Z}[x] /\left(x^{n}=0\right)$.

Since $N$ is a closed geodesic submanifold of $N_{i}$ of codimension 2 , by Theorem $1.9 N$ is connected. We now apply Theorem 1.11 and conclude that the inclusion $i: N \hookrightarrow N_{1}$ is $(2 n-4)$-connected. Let $[N]$ (resp. $\left.\left[N_{1}\right]\right)$ denote the fundamental class of $N$ (resp. $\left.N_{1}\right)$. Since $\operatorname{dim}(N)=2 n-4$, it is clear that $i_{*}([N])$ generates $H_{2 n-4}\left(N_{1}\right)$.

Let $x=P D\left(i_{*}([N])\right) \in H^{2}\left(N_{1}\right)$ be the Poincaré dual of $i_{*}([N])$. It is standard to verify the composition homomorphism

$$
H^{j}\left(N_{1}\right) \stackrel{i^{*}}{\rightarrow} H^{j}(N) \stackrel{\cap[N]}{\longrightarrow} H_{2 n-4-j}(N) \stackrel{i_{*}}{\rightarrow} H_{2 n-4-j}\left(N_{1}\right) \stackrel{\cap\left[N_{1}\right]}{\longrightarrow} H^{j+2}\left(N_{1}\right)
$$

is equal to $\cup x$, where $\cap$ (resp. $\cup$ ) is the cap (resp. cup) product. Recall that $\cap[N]$ (resp. $\left.\cap\left[N_{1}\right]\right)$ is the Poincaré dual map of $N$ (resp. $N_{1}$.)

Since $i: N \rightarrow N_{1}$ is a $(2 n-4)$-equivalence, the homomorphism $H^{j}\left(N_{1}\right) \stackrel{i^{*}}{\rightarrow}$ $H^{j}(N)$ is an isomorphism for $j<2 n-4$ and an injection for $j=2 n-4$. Similarly, $H_{2 m-4-j}(N) \stackrel{i_{*}}{\longrightarrow} H_{2 n-4-j}\left(N_{1}\right)$ is an isomorphism for $j>0$ and surjection for $j=0$. Therefore the above homomorphism $\cup x: H^{j}\left(N_{1}\right) \rightarrow H^{j+2}\left(N_{1}\right)$ is an isomorphism for $0<j<2 m-4$. Therefore $H^{2 m-1}\left(N_{1}\right)=0$ for all $1 \leq 2 n-1 \leq 2 n-3$. By the universal coefficients theorem $H_{*}\left(N_{1}\right)$ is torsion free.

If $x=0$, then $\cup x=0$ and the above shows easily that $N_{1}$ is a homotopy sphere.

If $x \neq 0$, by the above $H^{2}\left(N_{1}\right) \cong H_{2}\left(N_{1}\right) \cong \mathbb{Z}$ is generated by $x$. The above isomorphism shows that $x^{n-2}$ generates $H^{2 n-4}\left(N_{1}\right)$. By Poincaré duality there is an element $y \in H^{2}\left(N_{1}\right)$ (since $\operatorname{dim} N_{1}=2 n-2$ ) such that $x^{n-2} \cup y\left[N_{1}\right]=1$. Clearly, we may write $y=d x$ for some integer $d$. Therefore $d= \pm 1$ and $x^{n-1}\left[N_{1}\right]= \pm 1$. This shows that the cohomology ring $H^{*}\left(N_{1}\right)$ is isomorphic to $\mathbb{Z}[x] /\left(x^{n}=0\right)$.

As a consequence of Proposition 2.3 and (4.2.2), we conclude a weak version of Problem 0.2.

Lemma 4.3. Let $M$ be closed simply connected $2 n$-manifold of positive sectional curvature $(n \geq 2)$. Assume $M$ admits an isometric $\mathbb{Z}_{p} \oplus \mathbb{Z}_{p}$-action $(p \geq p(n)$ ) with fixed point set codimension 4 . Then $M$ is homotopic to a sphere or a complex projective space.

Proof. Note that for $n=2$, Lemma 4.3 is included in Proposition 2.3. Hence, we can assume that $n \geq 3$.

Let $N \subseteq \operatorname{Fix}\left(M, \mathbb{Z}_{p} \oplus \mathbb{Z}_{p}\right)$ be a fixed point component of codimension 4 . From the isotropy representation, it is clear that there are two $\mathbb{Z}_{p}$ subgroups, $H_{1}$ and $H_{2}$, whose fixed point components $N_{i}$ contain $N$ and are both of codimension 2. By now Lemma 4.3 follows from (4.2.2).

The following is a consequence of Lemma 2.1 and Lemma 3.1. 
Corollary 4.4. Let $N^{n}$ be a closed manifold of positive sectional curvature.

(4.4.1) If $n$ is even and $\mathbb{Z}_{p}^{k}(p \geq p(n))$ acts isometrically on $N$, then $n \geq 2 k$.

(4.4.2) If $n$ is odd and $S^{1} \times \mathbb{Z}_{p}^{k}(p \geq p(n))$ acts isometrically on $N$, then $n \geq 2 k+1$.

Proof of Theorem $C$.

Observe that without loss of the generality, we may assume $k=\left[\frac{3 n}{4}\right]+1$ or 2 . By Theorem 1.2 it suffices to show $M$ is homotopic to a sphere or a complex projective space.

From the isotropy representation at a $\mathbb{Z}_{p}^{k}$-fixed point (see Lemma 2.1), it is easy to see that the $\mathbb{Z}_{p}^{k}$-action has an isotropy group $H \cong \mathbb{Z}_{p}$. Hence, we can assume an $H$-fixed point component $N$ such that on which $\mathbb{Z}_{p}^{k-1}=\mathbb{Z}_{p}^{k} / H$ acts effectively. By (4.4.1), $2(k-1) \leq \operatorname{dim}(N) \leq \operatorname{dim}(M)-2$.

We shall proceed by induction on $n$. It turns out that in order to apply the inductive assumption (see (4.6)), we need to check the first four cases $7 \leq n \leq 10$.

Case 1. Assume $\operatorname{dim}(M)=14$ and thus $k=\left[\frac{3 \cdot 7}{4}\right]+1=6$. Then $\operatorname{dim}(N)=10$ or 12 and $\mathbb{Z}_{p}^{5} \cong \mathbb{Z}_{p}^{6} / H$ acts effectively on $N$. If $\operatorname{dim}(N)=10$, then by Theorem A, $N$ is homeomorphic to $S^{10}$ or $\mathbb{C} P^{5}$. By Theorem 1.11, $N \hookrightarrow M$ is 7 -connected. Applying Lemma 4.1 to $(M, N)$, we conclude that $M$ is homotopic to $S^{14}$ or $\mathbb{C} P^{7}$.

If $\operatorname{dim}(N)=12$, by Theorem $1.11 N \hookrightarrow M$ is 11 -connected. By Lemma 4.1 it suffices to prove that $N$ is homotopic to $S^{12}$ or $\mathbb{C} P^{6}$.

Consider $\left(N, \mathbb{Z}_{p}^{5}\right)$. First, $\mathbb{Z}_{p}^{5}$ has a subgroup $H_{1}$ with a fixed point component $N_{1}$ of dimension equals to 8 or 10 and $\mathbb{Z}_{p}^{4} \cong \mathbb{Z}_{p}^{5} / H_{1}$ acts effectively on $N_{1}$. If $\operatorname{dim}\left(N_{1}\right)=8$, then by Theorem, A $N_{1}$ is homeomorphic to $S^{8}$ or $\mathbb{C} P^{4}$. By Theorem $1.11, N_{1} \hookrightarrow N$ is 5 -connected. By Lemma 4.1, we then conclude that $N$ is homotopic to $S^{12}$ or $\mathbb{C} P^{6}$.

If $\operatorname{dim}\left(N_{1}\right)=10$, then we are in a situation that $\left(M, \mathbb{Z}^{6}\right)$ has a $\mathbb{Z}_{p} \oplus \mathbb{Z}_{p}$-subgroup whose fixed point set has codimension 4 . We then conclude the desired result from Lemma 4.3.

Case 2. Assume $\operatorname{dim}(M)=16$ and thus $k=7$. Then $\operatorname{dim}(N)=12$ or 14 . If $\operatorname{dim}(N)=12$, then by Theorem $\mathrm{A}, N$ is homeomorphic to $S^{12}$ or $\mathbb{C} P^{6}$. By Theorem 1.11, $N \hookrightarrow M$ is 9-connected. Now Lemma 4.1 applies to conclude that $M$ is homotopic to $S^{16}$ or $\mathbb{C} P^{8}$.

If $\operatorname{dim}(N)=14$, then by Case 1 we then conclude that $N$ is homotopic to $S^{14}$ or $\mathbb{C} P^{7}$. By Theorem 1.11 and Lemma 4.1, we then conclude that $M$ is homotopic to $S^{16}$ or $\mathbb{C} P^{8}$.

Case 3. Assume $\operatorname{dim}(M)=18$ and thus $k=\left[\frac{3 \cdot 9}{4}\right]+2=8$. Then $\operatorname{dim}(N)=14$ or 16. If $\operatorname{dim}(N)=14$, then by Theorem A $N$ is homeomorphic to $S^{14}$ or $\mathbb{C} P^{7}$. By Theorem 1.11, $N \hookrightarrow M$ is 11-connected. Now Lemma 4.1 applies to conclude that $M$ is homotopic to $S^{18}$ or $\mathbb{C} P^{9}$.

If $\operatorname{dim}(N)=16$, then by Case 2 we then conclude that $N$ is homotopic to $S^{16}$ or $\mathbb{C} P^{8}$. By (4.2.1) and Theorem 1.11, we then conclude that $M$ is homotopic to $S^{18}$ or $\mathbb{C} P^{9}$.

Case 4. Assume $\operatorname{dim}(M)=20$ and thus $k=\left[\frac{3 \cdot 10}{4}\right]+2=9$. Then $\operatorname{dim}(N)=16$ or 18. If $\operatorname{dim}(N)=16$, then by Theorem $\mathrm{A}, N$ is homeomorphic to $S^{14}$ or $\mathbb{C} P^{7}$. By Theorem 1.11, $N \hookrightarrow M$ is 13 -connected. Now Lemma 4.1 applies to conclude that $M$ is homotopic to $S^{20}$ or $\mathbb{C} P^{10}$.

If $\operatorname{dim}(N)=18$, then by Case 3 we then conclude that $N$ is homotopic to $S^{16}$ or $\mathbb{C} P^{8}$. By Theorem 1.11 and Lemma 4.1, we then conclude that $M$ is homotopic 
to $S^{20}$ or $\mathbb{C} P^{10}$.

We now proceed the rest of the proof by induction on $\operatorname{dim}(M)=2 n$ starting with $n=10$. Assume that Theorem $\mathrm{C}$ holds for $n \geq 10$.

Consider $\operatorname{dim}(M)=2(n+1)$.

a. Assume $n \neq 0(\bmod 4)$ (i.e., $n+1 \neq 1(\bmod 4))$. Then $k=\left[\frac{3(n+1)}{4}\right]+1=$ $\left[\frac{3 n}{4}\right]+2$. As in the above, there is a subgroup $H$ isomorphic to $\mathbb{Z}_{p}$ with a fixed point component $N$ satisfying

$$
14 \leq 2\left(\left[\frac{3 n}{4}\right]+1\right) \leq \operatorname{dim}(N) \leq 2 n \quad(n \geq 9)
$$

since $\mathbb{Z}_{p}^{\left[\frac{3 n}{4}\right]+1} \cong \mathbb{Z}^{\left[\frac{3 n}{4}\right]+2} / H$ acts effectively on $N$.

If $n \neq 1,2(\bmod 4)$, by $(4.5)$ we can apply the inductive assumption to $\left(N, \mathbb{Z}_{p}^{\left[\frac{3 n}{4}\right]+1}\right)$ and conclude that $N$ is homotopic to a sphere or a complex projective space. Since $\operatorname{dim}(N) \geq 2\left[\frac{3 n}{4}\right]+2 \geq n+2,[2 \operatorname{dim}(N)-2(n+1)+1] \geq n+3$. Hence, by Theorem $1.11 N \hookrightarrow M$ is at least $(n+3)$-connected. By Lemma 4.1 we conclude that $M$ is homotopic to $S^{2(n+1)}$ or $\mathbb{C} P^{n+1}$.

If $n=1$ or $2(\bmod 4)$, then $\left(N, \mathbb{Z}_{p}^{\left[\frac{3 n}{4}\right]+1}\right)$ has an isotropy group $H_{1}$ isomorphic to $\mathbb{Z}_{p}$ with a fixed point component $N_{1}$ satisfying

$$
14 \leq 2\left[\frac{3 n}{4}\right] \leq \operatorname{dim}\left(N_{1}\right) \leq \operatorname{dim}(N)-2 \leq 2(n-1) \quad(n \geq 10)
$$

If $\operatorname{dim}\left(N_{1}\right) \leq 2(n-2)$, then $\left[\frac{3 n}{4}\right] \geq\left[\frac{3(n-2)}{4}\right]+1$. By (4.6) we can apply the inductive assumption to $\left(N_{1}, \mathbb{Z}_{p}^{\left[\frac{3 n}{4}\right]}\right)$ to conclude that $N_{1}$ is homotopic to a sphere or a complex projective space, and therefore by Lemma 4.1, $N$ is homotopic to a sphere or a complex projective space. Similarly, $M$ is homotopic to $S^{2(n+1)}$ or $\mathbb{C} P^{n+1}$.

If $\operatorname{dim}\left(N_{1}\right)=2(n-1)$, then we are in a situation that $\left(M, \mathbb{Z}_{p}^{\left[\frac{3 n}{4}\right]+2}\right)$ has a $\mathbb{Z}_{p} \oplus \mathbb{Z}_{p}$ subgroup of fixed point set codimension 4 . We then conclude the desired result by applying Lemma 4.2 .

b. Assume $n=0(\bmod 4)$. Then $\left[\frac{3(n+1)}{4}\right]+1=\frac{3 n}{4}+1$. Since $n \neq 1,2(\bmod 4)$, we can apply the inductive assumption to conclude that $N$ is homotopic to a sphere or a complex projective space. Since $\operatorname{dim}(N) \geq 2\left(\left[\frac{3 n}{4}\right]+1\right) \geq n+4$ (because $n \geq 10$ ), $[2 \operatorname{dim}(N)-2(n+1)+1] \geq n+3$. Hence, by Theorem $1.11 N \hookrightarrow M$ is at least $(n+3)$-connected. Once again by Lemma 4.1 , we conclude that $M$ is homotopic to $S^{2(n+1)}$ or $\mathbb{C} P^{n+1}$.

Proof of Theorem D.

We shall follow the strategy in the proof of Theorem C. By Theorem 1.2, it suffices to show that $M$ is a homotopy sphere.

We first observe that by Sublemma 3.2, there is an $H$-fixed point component $N$ with $H \cong \mathbb{Z}_{p}$ and $\left(S^{1} \times \mathbb{Z}_{p}^{k}\right) / H$ acts effectively on $N$. By (4.4.2), $2(k-1)+1 \leq$ $\operatorname{dim}(N) \leq \operatorname{dim}(M)-2$.

Case 1. Assume $\operatorname{dim}(M)=2 \cdot 7+1=15$ and thus $k=\left[\frac{3 \cdot 7}{4}\right]+1=6$. From the above, $\operatorname{dim}(N)=11$ or 13 and $S^{1} \times \mathbb{Z}_{p}^{6} / H$ acts effectively on $N$. If $\operatorname{dim}(N)=13$, then by (4.2.1) $M$ is homeomorphic to $S^{15}$. If $\operatorname{dim}(N)=11$, then by Theorem 
B, $N$ is homeomorphic to $S^{11}$ (note that in this case, $\left(S^{1} \times \mathbb{Z}_{p}^{6}\right) / H \cong S^{1} \times \mathbb{Z}_{p}^{5}$ ). By Theorem 1.11, $N \hookrightarrow M$ is 8-connected. Hence, by the Poincaré duality $M$ is homotopic to $S^{15}$.

Case 2. Assume $\operatorname{dim}(M)=17$ and $k=7$. Then $\operatorname{dim}(N)=13$ or 15 and $\left(S^{1} \times\right.$ $\left.\mathbb{Z}_{p}^{6}\right) / H$ acts effectively on $N$. If $\operatorname{dim}(N)=15$, then by (4.2.1) $M$ is homeomorphic to $S^{17}$. If $\operatorname{dim}(N)=13$, then by Theorem B $N$ is homeomorphic to $S^{13}$. By Theorem 1.11, $N \hookrightarrow M$ is 10-connected. By the Poincaré duality $M$ is homotopic to $S^{17}$.

We now proceed the rest of the proof by induction on $\operatorname{dim}(M)=2 n+1$ starting with $n=8$.

Consider $\operatorname{dim}(M)=2(n+1)+1(n \geq 8)$.

a. Assume $n+1=1(\bmod 4)$ and thus $k=\left[\frac{3(n+1)}{4}\right]+2$.

Note that $\left(S^{1} \times \mathbb{Z}_{p}^{\left[\frac{3(n+1)}{4}\right]+2}\right) / H$ contains a subgroup isomorphic to $S^{1} \times \mathbb{Z}_{p}^{\left[\frac{3(n+1)}{4}\right]+1}$. Since $n \geq 8$,

$$
15 \leq 2\left(\left[\frac{3(n+1)}{4}\right]+1\right)+1 \leq \operatorname{dim}(N) \leq 2 n+1 .
$$

If $\operatorname{dim}(N)=2 n+1$, then by (4.2.1) $M$ is homeomorphic to a sphere. Note that

$$
\left[\frac{3(n+1)}{4}\right]+1 \geq\left[\frac{3(n-1)}{4}\right]+2 .
$$

If $\operatorname{dim}(N) \leq 2(n-1)+1$, then by $(4.7)$ and Case 1 we can apply the inductive assumption and conclude that $N$ is homotopic to a sphere. Since

$$
2 \operatorname{dim}(N)-(2(n+1)+1)+1 \geq 4\left[\frac{3(n+1)}{4}\right]+2-(2(n+1)+1)+1=n+3,
$$

by Theorem 1.11, $N \hookrightarrow M$ is at least $(n+3)$-connected. Hence, by the Poincaré inequality, $M$ is also homotopic to a sphere.

b. Assume $n+1 \neq 1(\bmod 4)$ and thus $k=\left[\frac{3(n+1)}{4}\right]+1$. Since $n \geq 9$ (note that $n=8$ is considered in a),

$$
15 \leq 2\left(\left[\frac{3(n+1)}{4}\right]+1\right)+1 \leq \operatorname{dim}(N) \leq 2 n+1,
$$

and $\left(S^{1} \times \mathbb{Z}_{p}^{\left[\frac{3(n+1)}{4}\right]+1}\right) / H$ acts effectively on $N$. If $\operatorname{dim}(N)=2 n+1$, then by $(4.2 .1)$ $M$ is homeomorphic to a sphere. Note that

$$
\left[\frac{3(n+1)}{4}\right] \geq\left\{\begin{array}{ll}
{\left[\frac{3(n-1)}{4}\right]+1} & n-1 \neq 1(\bmod 4) \\
{\left[\frac{3(n-1)}{4}\right]+2} & n-1=1(\bmod 4)
\end{array} .\right.
$$

If $\operatorname{dim}(N) \leq 2(n-1)+1$, then by $(4.8)$ and Case 1 we can apply the inductive assumption and conclude that $N$ is homeomorphic to a sphere. Since

$$
2 \operatorname{dim}(N)-(2(n+1)+1)+1 \geq 4\left[\frac{3(n+1)}{4}\right]+2-(2(n+1)+1)+1=n+3,
$$

by Theorem $1.11 N \hookrightarrow M$ is at least $(n+3)$-connected. Hence, by the Poincaré duality $M$ is also homotopic to a sphere. 


\section{REFERENCES}

[AW] S. Aloff; N. R. Wallach, An infinite family of 7-manifolds admitting positive curved Riemannian structures, Bull. Amer. Math. Soc. 81 (1975), 93-97.

[Ba1] Y. V. Bazaikin, On a family of compact 13-dimensional manifold with positive curvature, Sibirsk. Mat. Zh. 37 (1996), 1219-1237.

[Ba2] Y. V. Bazaikin, A manifold with positive sectional curvature and fundamental group $\mathbb{Z}_{3} \oplus \mathbb{Z}_{3}$, Sibirsk. Mat. Zh. 40 (1999), 994-996.

[Be] M. Berger, Les Varietes Riemanniennes homogènes normales simplement coonnexes à courbure strictement positive, Ann. Scuola Norm. Sup. Psia 15 (1961), 179-246.

[Ber] L. B. Bergery, Les variètès Riemanniennes homogènes simplement connexes de dimension impair a courture strictement positive, J. Math. Pures Appl. 55 (1976), 47-68.

[Br] G. Bredon, Introduction to compact transformation groups, Academic Press 48 (1972).

[BGP] Y. Burago; M. Gromov; Perel'man, A.D. Alexandov spaces with curvature bounded below, Uspekhi Mat. Nauk. 47:2 (1992), 3-51.

[Es] J.-H Eschenburg, New examples of manifolds with strictly positive curvature, Invent. Math. 66 (1982), 469-480.

[FMR] F. Fang; S. Mendonca; X. Rong, A connectedness principle in the geometry of positive curvature, Preprint (2001).

[FR1] F. Fang; X. Rong, Topology of positively curved manifolds of almost maximal symmetry rank, Preprint.

[FR2] F. Fang; X. Rong, Positively curved manifolds of almost maximal symmetry rank in dimensions 6,7 , In preparation.

[Fr] M. Freedman, Topology of Four Manifolds, J. of Diff. Geom. 28 (1982), 357-453.

[Gr] M. Gromov, Curvature, diameter and Betti numbers, Commentarii Math. Helvetici 56 (1981), 179-195.

[Gro] K. Grove, Geometry of, and via symmetries, Univ. Lecture Ser., Amer. Math. Soc., Providence, RT 27 (2002), 31-53.

[GS] K. Grove; C. Searle, Positively curved manifolds with maximal symmetry-rank, J. Pure Appl. Alg. 91 (1994), 137-142.

[GSh] K. Grove; K. Shankar, Rank two fundamental groups of positively curved manifolds, J. Geom. Anal. 10 (2000), 679-682.

[Ha] R. Hamilton, Three-manifolds with positive Ricci curvature, J. Diff. Geom. 17 (1982), 255-306.

[Hi] A. Hicks, Group actions and the topology of nonnegatively curved 4 manifolds, Illinois J. Math. 41 (1997), 421-437.

[Hs] W. Hsiang, Cohomology theory of topological transformation groups, Ergebnisse der Mathematik und inere Grenzgebiete 85 (1975).

[HK] W. Hsiang; B. Kleiner, On the topology of positively curved 4-manifolds with symmetry, J. Diff. Geom. 30 (1989), 615-621.

[Ko] S. Kobayashi, Transformation groups in differential geometry, Springer-Verlag Berlin Heidelberg New York (1972).

[Pe] P. Petersen, Comparison geometry problem list, Fields Inst. Monogr. 4 (1993), 87-115.

[Pet] A. Petrunin, Parallel transportation for Alexandrov space with curvature bounded below, Geom. Funct. Anal. 8 (1998), 123-148.

[PS] T. Püttmann; C. Searle, The Hopf conjecture for manifolds with low cohomogeneity or high symmetry rank, Proc. Amer. Math. Soc. 130 (2002), 163-166.

[Ro1] X. Rong, Positive curvature, local and global symmetry, and fundamental groups, Amer. J. Math. 121 (1999), 931-943.

[Ro2] X. Rong, Positively curved manifolds with almost maximal symmetry rank, Geometriae Dedicata 59 (2002), 157-182.

[Sh] K. Shankar, On the fundamental group of positively curved manifolds, J. Diff. Geom. 49 (1998), 179-182.

[Sm] S. Smale, Generalized Poincaré conjecture in dimension > 4, Ann. of Math. 74 (1961), 391-406.

[Su] D. Sullivan, Triangulating homotopy equivalences and homeomorphisms, Geometric Topology Seminar Notes, in "The Hauptvermutung Book" , A collection of papers on the topol- 
ogy of manifolds, K-monographs in Math. 1 (edited by A. Ranicki, Kluwer Academic Publishers, 1995).

[Wa] N. R. Wallach, Compact homogeneous manifolds with strictly positive curvature, Ann. of Math. 96 (1972), 277-295.

[Wi] B. Wilking, Torus actions on manifolds of positive sectional curvature, preprint (August 2002).

[Ya] D. Yang, On the topology of nonnegatively curved simply connected 4-manifolds with discrete symmetry, Duke Math. J. 74 (1994), 531-545.

Nankai Institute of Mathematics, Nankai University, Tianjing 300071, P.R.China. Instituto de Matematica, Universidade Federal Fluminense, Niteroi, RJ 24005, Brasil E-mail address: ffang@nankai.edu.cn fuquan@mat.uff.br

Mathematics Department, Capital Normal University, Beijing, P.R.C.

- Mathematics Department, Rutgers University, New Brunswick, NJ 08903, U.S.A

E-mail address: rong@math.rutgers.edu 